\title{
Épidémiologie descriptive des cas décédés de COVID-19 signalés lors de la première vague de l'épidémie au Canada, du 15 janvier au 9 juillet 2020
}

\author{
Équipe de surveillance et d'épidémiologie de la COVID-19¹ de l'Agence de la santé publique du \\ Canada
}

\section{Résumé}

Cette communication rapide décrit les décès parmi les cas de maladie à coronavirus 2019 (COVID-19) au Canada par province et territoire et par caractéristiques des cas. Sur les 106804 cas de COVID-19 signalés au Canada en date du 9 juillet 2020, 8749 ont entraîné la mort, ce qui représente un taux de mortalité de 23,3 pour 100000 habitants et un taux de létalité de $8,2 \%$. Au Canada, le taux de létalité varie de $0 \%$ à $10 \%$ selon les provinces et les territoires, les différences reflétant probablement les différences d'ampleur de l'épidémie dans chaque juridiction, ainsi que le lieu et les victimes des épidémies localisées (e.g. les éclosions dans les établissements de soins de longue durée, qui touchent les personnes âgées souffrant de multimorbidités). Le taux de létalité augmentait avec l'âge et le nombre de problèmes médicaux préexistants, ainsi que parmi les résidents des établissements de soins de longue durée et des foyers de personnes âgées. Des plans sont en cours pour recueillir des renseignements plus détaillés sur les cas, y compris la race et l'ethnicité, ce qui nous permettra de mieux comprendre les communautés les plus touchées par la COVID-19. Des études sur la surmortalité, une mesure du nombre de personnes qui sont mortes de n'importe quelle cause par rapport à la moyenne historique, permettront de clarifier l'impact complet de la COVID-19 au sein des juridictions canadiennes.
Cette oeuvre est mise à la disposition selon les termes de la licence internationale Creative Commons Attribution 4.0

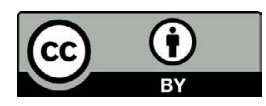

Affiliation

${ }^{1}$ Agence de la santé publique du Canada, Ottawa, ON

Correspondance :

lindsay.whitmore@canada.ca

Citation proposée : Équipe de surveillance et d'épidémiologie de la COVID-19 de l'Agence de la santé publique du Canada. Épidémiologie descriptive des cas décédés de COVID-19 signalés lors de la première vague de l'épidémie au Canada, du 15 janvier au 9 juillet 2020. Relevé des maladies transmissibles au Canada 2020;46(10):388-93. https://doi.org/10.14745/ccdr.v46i10a06f

Mots-clés : COVID-19, Canada, mortalité, épidémiologie, taux de létalité

\section{Introduction}

En début juillet 2020, le Canada avait réussi à aplatir la vague initiale de la pandémie de maladie à coronavirus 2019 (COVID-19), ramenant le nombre moyen de cas quotidiens déclarés d'un pic de 1600 au cours de la dernière semaine d'avril à 290 cas dans la semaine précédant le 9 juillet 2020 . Tragiquement, au cours de cette première vague, le Canada a enregistré le $20^{\mathrm{e}}$ taux brut de mortalité le plus élevé au monde au 9 juillet 2020 (1). Cette communication rapide décrit les décès parmi les cas de COVID-19, en utilisant les données disponibles signalées au cours de cette première vague du $1^{\text {er }}$ janvier 2020 au 9 juillet 2020, afin d'éclairer les mesures visant à minimiser les décès dus à la COVID-19 à l'avenir.

\section{Situation actuelle}

En date du 9 juillet 2020, 106804 cas de COVID-19 ont été déclarés au Canada, et le nombre de décès déclarés parmi ces cas était de 8749 . Le nombre de décès déclarés quotidiennement a augmenté régulièrement depuis la fin mars pour atteindre un pic au début mai; en moyenne, 177 décès ont été déclarés quotidiennement entre le 30 avril et le 6 mai. Le nombre quotidien de décès déclarés a ensuite diminué régulièrement et, dans la semaine précédant le 9 juillet 2020, une moyenne de 15 décès a été déclarée chaque jour (figure 1). Cela correspond à la tendance du nombre de cas déclarés quotidiennement qui a été observée deux à trois semaines plus tôt. Cette situation était attendue, puisque les décès liés 
à la COVID-19 représentent des infections survenues plusieurs semaines auparavant (2-4).

\section{Figure 1 : Décès quotidiens signalés parmi les cas de COVID-19 au Canada et moyenne mobile sur sept jours, du 8 mars au 9 juillet $2020(N=8749)^{a}$}

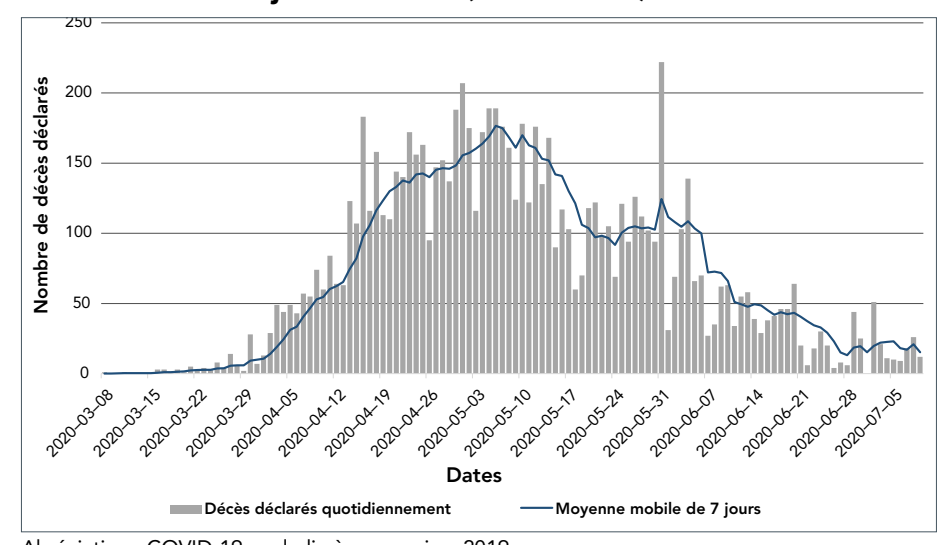

Abréviation : COVID-19, maladie à coronavirus 2019

a La forte augmentation du 31 mai 2020 est un artefact de déclaration, et est due au fait que le Québec a déclaré des décès qui avaient été précédemment identifiés, mais non déclarés

Le taux de mortalité brut du Canada au 9 juillet 2020 était de 23,3 décès liés à la COVID-19 pour 100000 habitants. En raison du délai entre la date de l'infection et la date du décès, le résultat clinique final pour les cas déclarés plus récemment n'est pas encore connu et, par conséquent, ce taux de mortalité a probablement sous-estimé le taux réel. Selon les données disponibles, le taux de mortalité de la COVID-19 au Canada était le $20^{\text {e }}$ plus élevé au monde, bien qu'il soit comparable ou inférieur à celui de plusieurs pays européens et nord-américains (1). Il est toutefois important de noter que le nombre de décès liés à la COVID-19 dans un pays est fondé sur plusieurs facteurs interdépendants, notamment la capacité du système de soins de santé, la façon dont les décès sont définis et saisis, le stade de l'épidémie et la répartition de la population d'un pays en fonction de la santé et de l'âge. Pour ces raisons, les comparaisons entre pays doivent être interprétées avec prudence.

Au 9 juillet 2020, le taux de létalité au Canada, calculé en divisant le nombre total de décès par le nombre de cas déclarés, était de 8,2\%. Comme pour le taux brut de mortalité, il s'agit probablement d'une mesure biaisée du véritable taux de létalité, puisque le résultat (i.e. les personnes qui se sont rétablies ou sont décédées) n'est pas encore connu pour les cas récemment déclarés.

Dans l'ensemble du Canada, le taux de létalité varie de $0 \%$ à $10 \%$ selon la province ou le territoire (tableau 1), et le taux de mortalité pour 100000 habitants canadiens varie de 0,3 à 66,1 (dans les provinces et territoires où il y a eu des décès). Après normalisation pour l'âge, qui a pris en considération des différences dans la répartition par âge entre les provinces, le taux de mortalité pour 100000 habitants se situait entre 0,2 et 60,4 . Après normalisation selon l'âge, les différences de taux de mortalité par province étaient probablement dues à plusieurs facteurs, dont le plus important était la propagation de la COVID-19 dans les établissements de soins de longue durée et les foyers pour personnes âgées. L'Ontario et le Québec affichent les taux de mortalité normalisés selon l'âge les plus élevés, soit 18,4 et 60,4 respectivement, plus de $70 \%$ de ces décès se sont produits dans les établissements de soins de longue durée et les résidences pour personnes âgées (tableau 1).

En effet, l'impact de la pandémie de COVID-19 sur les résidents des établissements de soins de longue durée et des résidences pour personnes âgées a été sévère. En date du 9 juillet 2020 (tableau 1), environ $81 \%$ des décès liés à la COVID-19 au Canada se sont produits parmi les résidents, l'Ontario et le Québec étant particulièrement touchés, avec respectivement 2011 et 4765 décès parmi les résidents d'établissements de soins de longue durée et de résidences pour personnes âgées. Cela peut être dû à la taille de la population, à sa densité et à l'ampleur de la transmission communautaire au sein de ces provinces particulières (6).

Sur les 106804 cas et 8749 décès déclarés au Canada le 9 juillet 2020, des renseignements plus détaillés ont été communiqués à l'Agence de la santé publique du Canada pour $106321(99,5 \%)$ cas et $8711(99,6 \%)$ décès à cette même date. Une analyse des décès liés à la COVID-19 selon l'âge, le sexe, les problèmes médicaux préexistants et les soins de longue durée et le statut de résident d'une résidence pour personnes âgées a été réalisée à l'aide de cet ensemble de données plus détaillées fournies par les provinces et les territoires.

Le taux de létalité était inférieur à $1 \%$ dans toutes les tranches d'âge jusqu'à 50 ans, puis augmentait rapidement avec l'âge, avec un taux de létalité de 1,2\% pour les 50 à 59 ans et jusqu'à $34,4 \%$ pour les 80 ans et plus (tableau 2). Les femmes canadiennes ont été globalement plus touchées, avec un taux de mortalité plus élevé pour 100000 (24,9 chez les femmes contre 21,3 chez les hommes). Cependant, parmi les cas, les hommes avaient un taux de létalité plus élevé que les femmes $(8,5 \%$ contre 7,9\%). Ces derniers résultats font écho à une différence entre les sexes observée parmi les cas de maladies graves dues à la COVID-19 dans d'autres pays $(7,8)$, ce qui peut refléter des différences entre les sexes dans la prévalence des problèmes médicaux préexistants et dans les comportements à risque comme le tabagisme (8).

En se fondant sur les renseignements limités disponibles sur les problèmes médicaux préexistants ( $n=6350)$, le taux de létalité a généralement augmenté au fur et à mesure que le nombre de problèmes médicaux préexistants augmentait, globalement et dans toutes les tranches d'âge (tableau 3). Cette augmentation a été marquée chez les personnes âgées de 60 à 79 ans, où le taux de létalité était de $3,1 \%$ chez les personnes sans problèmes médicaux préexistants et de $25,5 \%$ chez les personnes ayant trois problèmes médicaux préexistants ou plus. Les problèmes médicaux préexistants évalués dans les rapports de cas fournis 
Tableau 1 : Décès parmi les cas de COVID-19 au Canada au 9 juillet 2020, par province et territoire

\begin{tabular}{|c|c|c|c|c|c|c|}
\hline Province/territoire & $\begin{array}{l}\text { Total des } \\
\text { cas }\end{array}$ & Décès ${ }^{a}$ & $\begin{array}{l}\text { Décès parmi les résidents } \\
\text { des établissements de } \\
\text { soins de longue durée } \\
\text { et des résidences pour } \\
\text { personnes âgées }\end{array}$ & $\begin{array}{l}\text { Taux de } \\
\text { létalité }\end{array}$ & $\begin{array}{l}\text { Décès pour } \\
100000 \\
\text { habitants }^{c, d}\end{array}$ & $\begin{array}{l}\text { Taux de } \\
\text { mortalité } \\
\text { standardisé } \\
\text { selon l'âge par } \\
100000^{c, e, f}\end{array}$ \\
\hline Québec & 56216 & 5609 & 4765 & $10,0 \%$ & 66,1 & 60,4 \\
\hline Ontario & 36348 & 2703 & 2011 & $7,4 \%$ & 18,6 & 18,4 \\
\hline Alberta & 8519 & 161 & 117 & $1,9 \%$ & 3,7 & 4,8 \\
\hline Colombie-Britannique & 3028 & 186 & 131 & $6,1 \%$ & 3,7 & 3,5 \\
\hline Nouvelle-Écosse & 1066 & 63 & 57 & $5,9 \%$ & 6,5 & 5,8 \\
\hline Saskatchewan & 813 & 15 & 2 & $1,8 \%$ & 1,3 & 1,3 \\
\hline Manitoba & 325 & 7 & 1 & $2,2 \%$ & 0,5 & 0,6 \\
\hline Terre-Neuve-et-Labrador & 261 & 3 & 0 & $1,2 \%$ & 0,6 & 0,5 \\
\hline Nouveau-Brunswick & 166 & 2 & 2 & $1,2 \%$ & 0,3 & 0,2 \\
\hline Île-du-Prince-Édouard & 33 & 0 & 0 & $0 \%$ & $\mathrm{ADC}^{9}$ & $A D C^{9}$ \\
\hline Yukon & 11 & 0 & 0 & $0 \%$ & ADC & $A D C^{9}$ \\
\hline Territoires du Nord-Ouest & 5 & 0 & 0 & $0 \%$ & ADCg & $A D C^{9}$ \\
\hline Nunavut & 0 & 0 & 0 & $0 \%$ & $A D C^{g}$ & $A D C^{9}$ \\
\hline Canadah & 106804 & 8749 & 7086 & $8,2 \%$ & 23,3 & $23,3^{3}$ \\
\hline
\end{tabular}

Abréviations : ADC, aucun décès calculé; COVID-19, maladie à coronavirus 2019

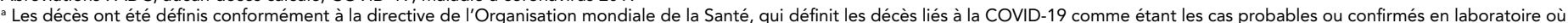

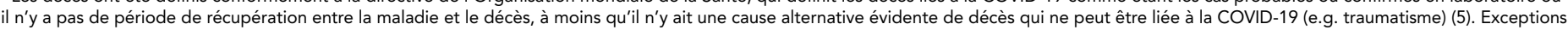

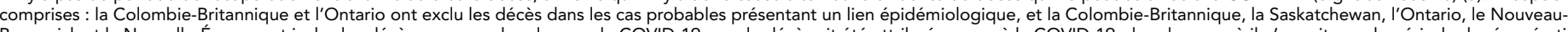

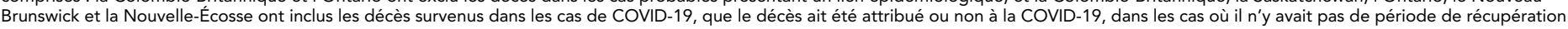
complète entre la maladie et le décès

b Sur la base des sites Web provinciaux et territoriaux, des points de presse, des analyses du Web, en date du 9 juillet 2020

' Le nombre de décès pour 100000 habitants n'a pas été calculé pour les provinces et territoires où il n'y a pas eu de décès

d Statistique Canada, estimations de la population au 1er juillet 2019

e Normalisation directe selon l'âge en utilisant les estimations de la population au 1er juillet 2019

' Le taux de mortalité standardisé selon l'âge pour 100000 habitants a été déterminé à l'aide des renseignements détaillés sur les cas reçus par l'Agence de santé publique du Canada pour 106198 cas et 8711 décès

9 Le nombre de décès pour 100000 habitants et le taux de mortalité standardisé selon l'âge pour 100000 habitants n'ont pas été calculés dans les juridictions où il n'y a pas eu de décès

${ }^{h}$ Le total pour le Canada comprend 13 cas identifiés chez des voyageurs rapatriés qui étaient en quarantaine en mars 2020. La mise à jour de leur statut n'était pas disponible

Le taux de mortalité standardisé selon l'âge pour 100000 habitants a été normalisé en fonction de la population nationale canadienne

Tableau 2 : Taux de létalité et de décès pour 100000 habitants, par âge et par sexe, au 9 juillet 2020 $(\mathrm{N}=106321)$

\begin{tabular}{|c|c|c|c|c|}
\hline $\begin{array}{c}\text { Caractéristiques } \\
\text { des cas }\end{array}$ & Cas & Décès & $\begin{array}{l}\text { Taux de } \\
\text { létalité }\end{array}$ & $\begin{array}{c}\text { Décès pour } \\
100000 \\
\text { habitants }^{\mathrm{a}}\end{array}$ \\
\hline \multicolumn{5}{|l|}{ Groupe d'âge ${ }^{b}$} \\
\hline $0-19$ ans & 7791 & 1 & $0,01 \%$ & 0,01 \\
\hline $20-29$ ans & 14813 & 8 & $0,05 \%$ & 0,2 \\
\hline $30-39$ ans & 14854 & 15 & $0,1 \%$ & 0,3 \\
\hline 40-49 ans & 16175 & 45 & $0,3 \%$ & 0,9 \\
\hline $50-59$ ans & 16140 & 200 & $1,2 \%$ & 3,8 \\
\hline $60-69$ ans & 10427 & 615 & $5,9 \%$ & 13,3 \\
\hline 70-79 ans & 7832 & 1570 & $20,1 \%$ & 54,7 \\
\hline 80 ans et plus & 18166 & 6257 & $34,4 \%$ & 385,1 \\
\hline Total & 106198 & 8711 & $8,2 \%$ & 23,0 \\
\hline \multicolumn{5}{|c|}{ Groupe d'âge par sexe $e^{b, c}$} \\
\hline \multicolumn{5}{|c|}{ Femme } \\
\hline $0-39$ ans & 19814 & 8 & $0,04 \%$ & 0,1 \\
\hline $40-59$ ans & 17827 & 94 & $0,5 \%$ & 1,9 \\
\hline $60-79$ ans & 9095 & 885 & $9,7 \%$ & 22,9 \\
\hline
\end{tabular}

Tableau 2 : Taux de létalité et de décès pour 100000 habitants, par âge et par sexe, au 9 juillet 2020 ( $N=106321$ ) (suite)

\begin{tabular}{|c|c|c|c|c|}
\hline $\begin{array}{c}\text { Caractéristiques } \\
\text { des cas }\end{array}$ & Cas & Décès & $\begin{array}{l}\text { Taux de } \\
\text { létalité }\end{array}$ & $\begin{array}{c}\text { Décès pour } \\
100000 \\
\text { habitants }^{a}\end{array}$ \\
\hline 80 ans et plus & 12457 & 3713 & $29,8 \%$ & 381,8 \\
\hline Total & 59193 & 4700 & $7,9 \%$ & 24,9 \\
\hline \multicolumn{5}{|l|}{ Homme } \\
\hline $0-39$ ans & 17519 & 16 & $0,1 \%$ & 0,2 \\
\hline $40-59$ ans & 14412 & 151 & $1,1 \%$ & 3,0 \\
\hline $60-79$ ans & 9117 & 1294 & $14,2 \%$ & 35,8 \\
\hline 80 ans et plus & 5596 & 2509 & $44,8 \%$ & 384,6 \\
\hline Total & 46644 & 3970 & $8,5 \%$ & 21,3 \\
\hline
\end{tabular}




\section{Tableau 3 : Taux de létalité par groupe d'âge parmi les cas de COVID-19 selon les problèmes médicaux préexistants et selon le statut de résident d'un établissement de soins de longue durée ou d'une résidence pour personnes âgées, 9 juillet 2020a}

\begin{tabular}{|c|c|c|c|c|c|c|c|c|}
\hline \multirow{4}{*}{$\begin{array}{c}\text { Caractéristiques } \\
\text { des cas }\end{array}$} & \multicolumn{8}{|c|}{ Décès/cas (taux de létalité) } \\
\hline & \multicolumn{8}{|c|}{ Âge (années) } \\
\hline & \multicolumn{2}{|c|}{$0-59$ ans } & \multicolumn{2}{|c|}{$60-79$ ans } & \multicolumn{2}{|c|}{80 ans et plus } & \multicolumn{2}{|c|}{ Total des cas } \\
\hline & $n / N$ & $\%$ & $n / N$ & $\%$ & $n / N$ & $\%$ & $n / N$ & $\%$ \\
\hline \multicolumn{9}{|c|}{ Nombre de problèmes médicaux préexistants $\mathrm{s}^{\mathrm{a}, \mathrm{b}}$} \\
\hline 0 & $4 / 4150$ & $0,1 \%$ & $32 / 1028$ & $3,1 \%$ & $67 / 224$ & $29,9 \%$ & $103 / 5402$ & $1,9 \%$ \\
\hline 1 & $2 / 135$ & $1,5 \%$ & $6 / 157$ & $3,8 \%$ & $12 / 55$ & $21,8 \%$ & $20 / 347$ & $5,8 \%$ \\
\hline 2 & $0 / 66$ & $0,0 \%$ & $18 / 141$ & $12,8 \%$ & $39 / 129$ & $30,2 \%$ & $57 / 336$ & $17,0 \%$ \\
\hline 3 et plus & $1 / 39$ & $2,6 \%$ & $26 / 102$ & $25,5 \%$ & $47 / 124$ & $37,9 \%$ & $74 / 265$ & $27,9 \%$ \\
\hline \multicolumn{9}{|c|}{ Résident d'un établissement de soins de longue durée ou de résidence pour personnes âgées ${ }^{c}$} \\
\hline Non & $6 / 8122$ & $0,1 \%$ & $34 / 1098$ & $3,1 \%$ & $35 / 171$ & $20,5 \%$ & $75 / 9391$ & $0,8 \%$ \\
\hline Oui & $3 / 33$ & $9,1 \%$ & $45 / 213$ & $21,1 \%$ & $155 / 512$ & $30,3 \%$ & $203 / 758$ & $26,8 \%$ \\
\hline
\end{tabular}

Abréviation : COVID-19, maladie à coronavirus 2019

a Les renseignements sur les problèmes médicaux préexistants n'étaient pas disponibles pour $94,03 \%(n=99971)$ des cas

${ }^{b}$ Les problèmes médicaux préexistants ont été définis comme suit : maladie cardiaque, trouble neurologique ou neuromusculaire chronique, diabète, maladie/condition d'immunodéficience, maladie du foie, malignité, maladie rénale et maladie respiratoire

'Les renseignements indiquant si le cas était ou non un résident d'un établissement de soins de longue durée ou d'une résidence pour personnes âgées n'étaient pas disponibles pour $90,45 \%$ $(\mathrm{n}=96$ 171) des cas

à l'Agence de la santé publique du Canada comprenaient les maladies cardiaques, les troubles neurologiques ou neuromusculaires chroniques, le diabète, les maladies/affections d'immunodéficience, les maladies du foie, les affections malignes, les maladies rénales et les maladies respiratoires. Le fait d'être résident d'un établissement de soins de longue durée ou d'une résidence pour personnes âgées a également entraîné un taux de létalité plus élevé dans l'ensemble $(26,8$ \%) et dans chaque groupe d'âge. Bien que les renseignements permettant de déterminer si le cas était ou non un résident d'un établissement de soins de longue durée ou d'une résidence pour personnes âgées soient limités ( $n=10150)$, ces résultats sont similaires à ceux d'autres études canadiennes, dans lesquelles le taux de létalité global parmi les résidents était estimé à $36 \%(6)$, et était supérieur à celui des canadiens âgés qui ne vivaient pas dans un tel environnement (Personal communication, D. Fisman et al.). Le taux de létalité plus élevé au sein de cette population reflète probablement un degré de fragilité et de multimorbidité chez les résidents qui augmente le risque de complications graves, y compris le décès (9).

Il y a de plus en plus de preuves que, comme aux États-Unis et au Royaume-Uni (10), les personnes appartenant à des minorités raciales et ethniques au Canada courent un risque accru de contracter la COVID-19 et de subir des complications graves, y compris le décès $(11,12)$. Au niveau national, il est prévu de commencer à l'automne 2020 la collecte de renseignements plus détaillés sur les cas, notamment sur la race, l'ethnicité et le statut socio-économique.

\section{Conclusion}

Les autorités de santé publique de tout le pays continueront à suivre de près le nombre de décès parmi les cas de COVID-19 afin d'orienter des mesures supplémentaires pour prévenir les issues fatales. Bien que les décès fournissent une indication tardive de la transmission de la COVID-19, ils donnent une indication claire de la gravité et de l'impact de la maladie et mettent en évidence la nécessité de protéger les populations vulnérables, notamment les personnes âgées et celles qui vivent avec de multiples problèmes médicaux préexistants. Plus particulièrement, les canadiens vivant dans des établissements de soins de longue durée et des résidences pour personnes âgées ont été gravement touchés, avec un taux de létalité plus élevé que celui des canadiens plus âgés qui ne vivent pas dans de tels établissements. En conséquence, les juridictions fédérales, provinciales et territoriales ont mis en œuvre de multiples mesures pour prévenir la transmission dans ces milieux. Ces mesures comprennent des lignes directrices actualisées sur la prévention et le contrôle des infections, et plus particulièrement en ce qui concerne les soins aux résidents d'établissements de soins de longue durée (13), des mesures politiques visant à garantir une dotation en personnel adéquate et à limiter les déplacements des travailleurs de la santé entre les établissements.

Le taux de mortalité par infection et la surmortalité sont d'autres mesures de la mortalité qui peuvent être utilisées pour décrire I'impact de la COVID-19 au Canada. Le taux de mortalité par infection est défini comme le nombre de décès divisé par le nombre d'individus infectés. Contrairement au taux de létalité, le taux de mortalité par infection n'est pas influencé par des facteurs tels que les changements de stratégie des tests de laboratoire. Des plans sont en cours pour mener des enquêtes sérologiques rapides pancanadiennes et régionales afin de déterminer l'étendue du coronavirus du syndrome respiratoire aigu sévère 2 (SRAS-CoV-2) dans la population et ces résultats permettront d'estimer le taux de mortalité par infection au Canada (14).

L'estimation de la surmortalité nous permettra de mieux comprendre l'impact de la pandémie de COVID-19 au-delà de la maladie elle-même. Par exemple, on peut s'attendre à une augmentation des décès dus à d'autres causes en raison des personnes que la peur de l'infection empêche de se faire soigner à temps. La surmortalité est la différence entre le nombre total de personnes qui sont décédées de quelque cause que ce soit au cours d'une période donnée et la moyenne historique pour la même période de l'année au cours des années précédentes. En juin 2020, Statistique Canada a publié un ensemble de données provisoires sur l'excès de décès de janvier à la semaine du 2 mai 2020, par rapport aux cinq années précédentes (15). Ces 
données étaient préliminaires et, à mesure que de plus amples renseignements seront disponibles pour toutes les juridictions canadiennes, nous comprendrons mieux l'impact réel de cette pandémie sur les canadiens.

\section{Déclaration des auteurs}

D. P. - Conceptualisation, projet original, révision et édition C. B. - Conceptualisation, conservation des données, analyse formelle, révision et édition

J. M. - Conservation des données, analyse formelle, révision et édition

L. W. - Révision et édition

A. C. - Conceptualisation, révision et édition

C. A. - Révision et édition

L. S. - Révision et édition

D. T. - Révision et édition

D. M. - Révision et édition

J. P. - Révision et édition

\section{Intérêts concurrents}

Aucun.

\section{Remerciements}

Les auteurs souhaitent remercier les partenaires de surveillance provinciaux et territoriaux, le Laboratoire national de microbiologie, les laboratoires de santé publique provinciaux partenaires et l'équipe de surveillance du Centre des opérations du portefeuille de la Santé de l'Agence de la santé publique du Canada.

L'équipe de surveillance et d'épidémiologie de la COVID-19 de I'Agence de santé publique du Canada comprenait D. Paquette, C. Bell, J. Macri, L. Whitmore, A. Currie, C. Archibald, L. Shah, D. Taylor, D. MacDonald et J. Pennock.

\section{Financement}

Ces travaux ont été soutenus par l'Agence de santé publique du Canada.

\section{Références}

1. Our World in Data. Statistics and Research. Coronavirus (COVID-19) Deaths (accédé 2020-07-24).

https://ourworldindata.org/covid-deaths
2. Zhou F, Yu T, Du R, Fan G, Liu Y, Liu Z, Xiang J, Wang Y, Song B, Gu X, Guan L, Wei Y, Li H, Wu X, Xu J, Tu S, Zhang Y, Chen $\mathrm{H}$, Cao B. Clinical course and risk factors for mortality of adult inpatients with COVID-19 in Wuhan, China: a retrospective cohort study [published correction appears in Lancet. 2020 Mar 28;395(10229):1038]. Lancet 2020 Mar;395(10229):1054-62. DOI PubMed

3. Chen T, Wu D, Chen H, Yan W, Yang D, Chen G, Ma K, Xu D, Yu H, Wang H, Wang T, Guo W, Chen J, Ding C, Zhang X, Huang J, Han M, Li S, Luo X, Zhao J, Ning O. Clinical characteristics of 113 deceased patients with coronavirus disease 2019: retrospective study. BMJ 2020 Mar;368:m1091. DOI PubMed

4. Verity R, Okell LC, Dorigatti I, Winskill P, Whittaker C, Imai N, Cuomo-Dannenburg G, Thompson $\mathrm{H}$, Walker PG, Fu H, Dighe A, Griffin JT, Baguelin M, Bhatia S, Boonyasiri A, Cori A, Cucunubá Z, FitzJohn R, Gaythorpe K, Green W, Hamlet A, Hinsley W, Laydon D, Nedjati-Gilani G, Riley S, van Elsland $S$, Volz E, Wang H, Wang Y, Xi X, Donnelly CA, Ghani AC, Ferguson NM. Estimates of the severity of coronavirus disease 2019: a model-based analysis. Lancet Infect Dis 2020 Jun;20(6):669-77. DOI PubMed

5. World Health Organization. Medical certification, ICD mortality coding, and reporting mortality associated with COVID-19. COVID-19: Surveillance, case investigation and epidemiological protocols. WHO; June 72020 (accédé 2020-07-05). https://www.who.int/publications/i/item/WHO2019-nCoV-mortality-reporting-2020-1

6. International Long Term Care Policy Network. Hsu AT, Lane N, Sinha SK, Dunning J, Dhuper M, Kahiel Z, Sveistrup $\mathrm{H}$. Understanding the impact of COVID-19 on residents of Canada's long-term care homes - ongoing challenges and policy responses (modifié 2020-0604). https://ltccovid.org/wp-content/uploads/2020/06/ LTCcovid-country-reports_Canada_June-4-2020.pdf

7. Jordan RE, Adab P, Cheng KK. Covid-19: risk factors for severe disease and death. BMJ 2020 Mar;368:m1198. DOl PubMed

8. Livingston E, Bucher K. Coronavirus Disease 2019 (COVID-19) in Italy. JAMA 2020 Apr;323(14):1335. DOI PubMed

9. Gardner W, States D, Bagley N. The Coronavirus and the Risks to the Elderly in Long-Term Care. J Aging Soc Policy 2020 Jul-Oct;32(4-5):310-5. DOI PubMed

10. Pan D, Sze S, Minhas JS, Bangash MN, Pareek N, Divall $P$, Williams CM, Oggioni MR, Squire IB, Nellums LB, Hanif W, Khunti K, Pareek M. The impact of ethnicity on clinical outcomes in COVID-19: A systematic review. EClinicalMedicine 2020 Jun;23:100404. DOI PubMed 
11. Agence ontarienne de protection et de promotion de la santé (Santé publique Ontario). Résumé épidémiologique amélioré. La COVID-19 en Ontario - un regard sur la diversitév: du 15 janvier 2020 au 14 mai 2020-Toronto (ON) : Imprimeur de la Reine pour l'Ontario, 2020. https:// www.publichealthontario.ca/-/media/documents/ncov/ epi/2020/06/covid-19-epi-diversity.pdf?la=fr

12. CBC News. Rocha R, Shingler B, Montpetit J. Montreal's poorest and most racially diverse neighborhoods hit hardest by COVID-19, data analysis shows. June 11, 2020. https://www.cbc.ca/news/canada/montreal/race-covid19-montreal-data-census-1.5607123

13. Gouvernement du Canada. Lignes directrices provisoires sur les soins aux résidents des établissements de soins de longue durée pendant la pandémie de COVID-19. Gouvernement du Canada (modifié 2020-07-17; accédé 2020-07-24). https://www.canada.ca/fr/sante-publique/ services/maladies/2019-nouveau-coronavirus/ document-orientation/residents-etablissement s-soins-longue-covid-19.html
14. Le Groupe de travail sur l'immunité face à la COVID-19. À propos (accédé 2020-07-05). https://www. covid19immunitytaskforce.ca/about

15. Statistique Canada. Nombre provisoire de décès et surmortalité, janvier à mars 2019 et janvier à mars 2020. Ottawa (ON) : Statistique Canada (accédé 2020-07-05). https://www150.statcan.gc.ca/n1/daily-quotidien/200513/ dq200513d-fra.htm

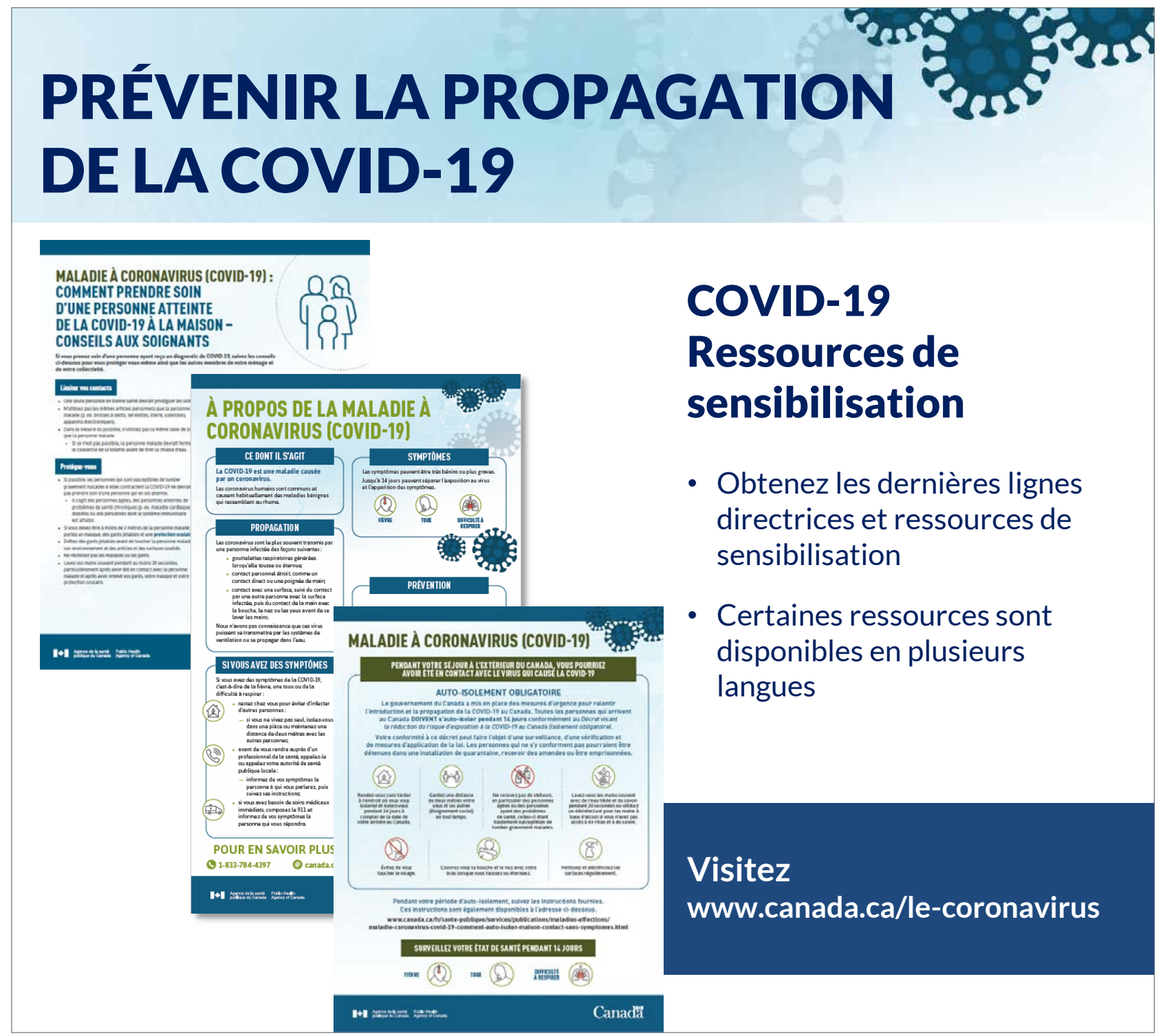

